\title{
A SIMULATION APPROACH TO ASSESS PARTNERS SELECTED FOR A COLLABORATIVE NETWORK
}

\author{
Andres, B. ; Poler, R. ; Camarinha-Matos, L. M. ${ }^{* *} \&$ Afsarmanesh, H. ${ }^{* *}$ \\ * Research Centre on Production Management and Engineering (CIGIP), Universitat Politècnica de \\ València (UPV). Calle Alarcón, 03801 Alcoy, Spain \\ ${ }^{* *}$ NOVA University of Lisbon - Faculty of Sciences and Technology, Portugal \\ ${ }^{* * * *}$ University of Amsterdam - Informatics Institute, Netherlands \\ E-Mail: bandres@cigip.upv.es, rpoler@cigip.upv.es, cam@uninova.pt, h.afsarmanesh@uva.nl
}

\begin{abstract}
Manufacturing enterprises are increasingly more aware of the importance of establishing collaborative relationships with their network partners, due to the advantages associated to collaboration. Nevertheless, the participation in a collaborative network $(\mathrm{CN})$ comes with associated challenges, namely the need to reduce the potential for conflicts among partners. A CN consists of heterogeneous partners, each one defining its own objectives and activating its own strategies. In this context, the ability to quickly identify partners with aligned strategies is crucial for smooth operation of the CN. The main aim of this paper is to address the partners' selection problem in the context of Virtual organizations Breeding Environments (VBE) that facilitate and enable the creation of Virtual Organisations (VO), as one type of CN. In a first stage, the sets of enterprises, characterised by having the required competencies to create the $\mathrm{VO}$, are identified among different potential candidates within the VBE. In a second stage, the strategies alignment approach, based on the system dynamics simulation method, is used for the partners' selection process, identifying the best set of enterprises. In this paper, the final stage of partners' selection process is addressed by obtaining the degree of alignment of the business strategies formulated by each set of enterprises. In the light of this, a system dynamics-simulation model, in AnyLogic, is presented to obtain the set of enterprises that have higher levels of alignment in its strategies. The proposed system dynamics-simulation model is applied to a case in the building industry, to deal with the partners' selection problem in a VBE with the aim of forming a stable and sustainable VO.

(Received in October 2016, accepted in March 2017. This paper was with the authors 1 month for 1 revision.)
\end{abstract}

Key Words: Collaborative Networks, System Dynamics, Partners' Selection, Strategies Alignment, Values Alignment, Trust

\section{INTRODUCTION}

The concept of collaborative network $(\mathrm{CN})$ has been widely studied over the last years motivated by the positive effects for the enterprises that collaborate [1]. In order to get a better understanding on effective ways of managing collaboration, various research studies have been developed [2, 3]. In the effort to consolidating CNs as a new discipline, [3] introduced the concept of collaborative network as "a network consisting of variety of autonomous, geographically distributed, and heterogeneous entities that collaborate to better achieve common or compatible goals". The growing research interest in collaboration led to the emergence of a wide variety of $\mathrm{CN}$ classes [3, 4], including extended enterprises, virtual enterprises (VE), virtual organisations (VO), virtual organization breeding environments (VBE), business ecosystems, non-hierarchical networks (NHN), etc. This paper focuses on $\mathrm{VO}$ creation in a VBE context. A VO is understood as " a set of independent organizations that share resources and skills to achieve its mission/goal" [3]. A VBE represents an association or cluster of organizations that "have both the potential and the will to cooperate with each other through the establishment of a long-term cooperation agreement and interoperable infrastructure" $[5,6]$. When a new business opportunity is identified by a VBE 
member, a subset of these organizations can be selected to constitute a VO [3]. The process of creating a VO requires a greater exchange of information and high commitment of all involved enterprises, shared responsibility, and the consideration of partners' objectives, strategies and value systems.

Participation in a VO has associated positive influences on the agility and responsiveness of the enterprises. Nevertheless, the creation of a VO, and therefore, the establishment of collaborative processes, raises a set of challenges [4]. Partners' selection is considered in the literature a major issue in the formation of a VO [7]. When selecting VO's partners, it must be guaranteed that they possess the necessary competencies to meet the business objectives of the VO. Moreover, Shamsuzzoha [8] defines different critical success factors, additional to the competencies, that determine the success of the selection of network partners, which include, trust, processes standardisation, knowledge and information sharing, compatible objectives, strategies and values, and availability of information and communication technologies (ICT). Due to the associated critical factors and their importance in the VO success, the partners' selection process has received much research attention along the years to achieve stable and sustainable collaborative relationships. In fact, finding the right partners in a VO is a difficult and time-consuming task, which negatively influences the agility of the VO formation process. For instance, overcoming misalignments resulting from the partners' heterogeneity (e.g. misalignments in ICT) requires large investment. Building trust, a major pre-requisite for any successful collaboration, is not straightforward, requiring considerable time. Therefore, partners" selection is not simply an "optimization" problem [9].

The heterogeneity of the enterprises participating in a VBE increases the likelihood of misalignments appearance, among the VO partners. Thus, although matching processes based on competencies are required as a basis $[10,11]$, many other factors need to be considered in the partners' selection process, some of them of a subjective nature. In addition to the criterion of competencies, the consideration of other filters is necessary, such as the assessment of trustworthiness [12], value systems alignment [13], or strategies alignment [14]. These "additional filters" provide complementary mechanisms to support partners' selection in a $\mathrm{CN}$ environment, reducing the potential for conflicts, and thus increasing $\mathrm{VO}$ sustainability. Towards this purpose, this article suggests the consideration of one additional filter, namely strategies alignment, to deal with the partners' selection process in a VBE context. The remaining of this paper is organised as follows: Section 2 presents a brief state of the art in the research area of VO, VBE, partners' selection, and complementary approaches to deal with the partners' selection collaborative process. Section 3 proposes an approach, based on the system dynamics-simulation method, to deal with the partners' selection, in order to obtain higher levels of alignment among strategies defined by the enterprises candidate to the VO. Section 4 presents a use case from construction industry, using the strategies alignment as an approach to assess partners selected for a VO. Finally, conclusions and future research lines are described in Section 5.

\section{STATE OF THE ART}

With the aim of giving the reader a better understanding of the target context, this section provides an overview of the involved research areas, as found in current literature. Complementary mechanisms to support partners' selection in a CN context are also identified, including trust assessment, value systems alignment, and strategies alignment.

\subsection{Virtual organizations Breeding Environment}

A VO Breeding Environment (VBE) is defined as "a long-term association of entities that are recruited from the open universe of organizations, and that prepare themselves to cooperate 
whenever an opportunity arises, adopting common operating principles and infrastructures, with the main goal of increasing both their chances and their preparedness towards collaboration in potential VO" $[5,6]$. VBE members are important actors during the phase of partners' selection for a VO. A variety of roles can be played by the actors in the VBE, among which we emphasize: (i) VBE member, for any organization that participates in the VBE activities; and (ii) VO Planner, which identifies and acquires new business opportunities, and identifies the necessary competencies, selects an appropriate set of partners, and structures the new VO. A VBE allows to (i) establish the base trust among the organizations to be involved in VOs, (ii) reduce the cost/time to find suitable partners for configuration of the VOs, and (iii) assist with the creation, establishment of agreements, and contract negotiation for establishment of VOs, thus reducing the risk of losses due to some organization failures $[3,5]$.

\subsection{Virtual Organisations}

One of the most addressed $\mathrm{CN}$ cases is the virtual organization (VO) and its particular case of virtual enterprise, which has increased its application domains, due to the possibility of rapidly creating a well-fit VO when a business opportunity appears, giving to the involved enterprises the required agility for survival in the global and rapidly changing current markets. A VO is defined in [3] as "a temporary organization triggered by a specific business / collaboration opportunity". The VO creation process is assumed to take place in the context of a VBE, and thus it is assumed to be fast. This assumption involves solving some important issues, such as identifying the potential partners or defining agreements on the roles, responsibilities and exploitation rights of each partner [9].

\subsection{Partners' selection approaches}

When a new business collaboration opportunity arises and the VO planner and decides structuring a new VO, it is needed to identify the necessary competencies, and select the partners that cover the competencies required by the VO. For this selection, manual or computer-assisted approaches are identified in the literature. As a starting point, the development of directories of enterprises' clusters, gathering information of enterprises profiles, products, services, resources and capabilities is often assumed [15]; in this regard, the Partners Search and Suggestion methodology [10,16] and the Rough VO planning approaches $[9,17]$ allow to determine a rough structure of the potential VO, identifying the required partners' competencies and capacities. Optimization-based methods started to be developed in the mid-1990s, considering the selection of partners through: (i) cost minimization models, (ii) multi-criteria models, and (iii) matching of skills and needs. Quantitative and qualitative analysis methods, are worth to mention, such as vague sets theory, to deal with uncertain information, or PSO (stochastic population-based optimization algorithm) [7]. Multi-criteria decision making problems, have been proposed as an approach to rank alternative VO configurations, using TOPSIS (a technique for ordering preferences by similarity to an ideal solution) [18]. Causal maps approach is used to quickly identify partners with compatible core-values to assist the selection of VO members [19]. The Analytical Hierarchical Process (AHP) has also been considered as a decision model [20]. Regarding competencies matching, a distinction between hard and soft competencies is made [11] in order to capture a behavioural perspective, such as openness, ability to exchange knowledge, negotiation capabilities, etc. Simulation approaches are also used to deal with the partners' evaluation in a supply chain [21]. On the other hand, agent-based models have been used to support the selection of the partners, in the stages of formation and reconfiguration of a $\mathrm{VO}$ [22]. Finally, performance measurement models have been proposed to select VO partners, involving both intra and inter-organisational strategic indicators [16]. In the end, the 
effectiveness of the proposed models, guidelines and tools mainly depend on the availability of adequate information about the partners of the VBE.

\subsection{Additional approaches to deal with partners' selection for a VO}

In the partners' selection process, the consideration of additional criteria, in addition to the competencies criteria are necessary to increase the VO sustainability and reduce the potential for conflicts during VO operation. Such additional filters may include trust assessment, value systems alignment, and strategies alignment.

Trust assessment: Collaboration in a VO requires a base level of trust among the involved organizations. Therefore, for the partners' selection problem, assessment of the trust worthiness level of potential candidates is considered an important mechanism [5,6]. For instance, Msanjilla and Afsarmanesh [12] address inter-organizational trust in VBEs as an enabler for efficiently managing it, as well as creating VOs constituting its trustworthy members as potential partners. A facilitation of the process of creating and launching VOs through smoothing the partners' selection processes is an advantage that can be gained when trust relationships among member organizations have been established and properly managed in the VBE.

Value System Alignment: CNs are formed by autonomous and heterogeneous entities, in which each entity possesses its own set of values. As such, conflicts among partners might emerge due to possible values misalignment. In this regard, the ability to quickly identify partners with strong values alignment represents a promising approach for the formation of successful VOs [19]. Let us assume that both the VO planner defines the core-value system that will guide the behaviour of the VO being created, and that the core-value system of each partner is also known. In this context, a core-values alignment measurement can be carried out in order to reduce the potential conflicts that could appear among the considered VO members and, thus, supports the partner selection process [13]. In order to reduce risks, a combination of value systems alignment and negotiation mechanisms are used in [23].

Strategies Alignment: Similar to the value system alignment, the strategies alignment assessment process is another important criterion to select partners for a VO. As mentioned, a $\mathrm{CN}$ is composed of autonomous partners, each one with its own objectives and business strategies. The diversity of strategies adopted by these enterprises may lead to conflicting situations among them, as contradictions between the formulated strategies and the objectives defined might emerge. These contradictions are likely to appear when the enacted business strategies in one enterprise negatively influence the objectives set by other enterprises of the network. The lack of coherence and concordance among the adopted strategies leads to a misalignment situation [24]. Strategies misalignments may affect the achievement of enterprises' objectives, reducing their performance levels, and influencing the wealth of the established collaborative relationships [14]. Let us assume that the VO planner defines the strategies related to a business opportunity, and each potential partner formulates its own strategies to achieve its own objectives. A strategies alignment assessment can be performed in order to identify potential strategies misalignments and assist the partners' selection process by considering the degree of alignment. A discussion of the combination of value systems alignment and strategies alignment can be found in [25]. So far, the application of the strategies alignment is considered a novel approach for partners' selection in VO. In this regard, the paper proposes a sound system dynamics-based model, a methodology and an integrated environment to support the partners' selection process considering the strategies alignment criterion. 


\section{SYSTEM DYNAMICS APPROACH FOR PARTNERS' SELECTION}

\subsection{System dynamics simulation to model the strategies alignment process}

Each of the enterprises belonging to a VBE is assumed to define a set of objectives and formulate strategies to achieve these objectives. On the one hand, an enterprise objective is a result to be achieved, towards which efforts are addressed, and which aims to improve or stabilize the effectiveness and efficiency of such enterprise; e.g. O1: Increase the product sales by $10 \%$; O2: Reduce the costs of the product by $5 \%$. On the other hand, a strategy consists of a set of planned actions that an enterprise should follow to achieve the defined objectives. A strategy aims to reach a goal through a pattern of action; e.g. S1: Increase the marketing activities on the product (related with O1), S2: Conduct negotiations with the supplier partner to reduce purchasing costs (related with $O 2$ ). It is assumed that each strategy has a cost of activation, and that enterprises have to decide which strategies to activate depending on the activation costs and the impact that each strategy has on the defined objectives. Therefore, when dealing with the decision of which partners to select, the VO planner must consider both the adjustment of partners' capacities and resources and the degree of strategies alignment among the partners of the VO. Focusing on the strategies alignment, the most appropriate partners to be selected will be the ones with higher levels of strategies alignment. For assessing the strategies alignment, intra and inter-enterprise influences must be considered. On one hand, intra-enterprise influences measure the influences between strategies and the objectives of the same enterprise. On the other hand, inter-enterprise influences appear when the strategies of one network enterprise positively/negatively influence the objectives of the other enterprises of the network. In this regard, strategies alignment is reached when the strategies activated by a specific partner, positively affect its own objectives, but also positively influence the objectives defined by the rest of collaborative partners and the objective of $\mathrm{VO}$, so that performance at network level is maximised.

To model the strategies alignment process a system dynamics (SD) simulation based model has been proposed in [24]. SD allows solving abstract models, such as the mathematical ones, using computer techniques to model and solve complex systems [26]. SD is intuitive enough to represent the VO complex system, which consists of different autonomous entities and where the decisions of one node may affect the other network nodes operation. Therefore, SD enables to understand the structure and dynamics of the VO. SD revolves around the concept of feedback and causality between observable variables. According to [27], SD examines the interaction between various functions within a system. The representation of the causal relationships among system components facilitates the understanding of their occurrence, allowing improving the interaction between them. In the proposed system-dynamics simulation based approach the strategies alignment process is characterised by the positive and negative flows generated among the components of the modelled system, and the causal relationships established between the defined objectives and the strategies selected by each enterprise of the network [24]. To illustrate the idea, let us consider the model of a VO. The casual diagram represented in Fig. 1 is focused on two enterprises $(i$ and $j$ ), in which lined arrows represent intra-enterprise influences, while doted arrows represent the inter-enterprise influences between the enterprises. The elements belonging to this system are listed in Table I. These elements are characterised by representing aggregate magnitudes of the overall system, modelling it as a whole.

In this case, each enterprise, in the VO, is assumed to be independent, and it defines a set of objectives, whose achievement is measured though KPIs (KPIixk); it also formulates strategies in order to achieve these objectives, represented by the decision variable $u_{-}$Sis and characterised by ti_Sis. The relationship between the strategies and the objectives is 
represented in order to identify the positive and negative influences that the activation of certain strategies exerts on the objectives attainment. The strategies' positive or negative influences affect the enterprises performance, and consequently the network performance. A system dynamics-based simulation model is built in order to solve the Strategies Alignment Model (SAM) [24] and identify the optimum values of the decision variables $\left(u \_S i s\right.$ and $t i \_$Sis) that maximise the performance level of the global network (KPI_GLOBAL). The proposed simulation model relies on a performance measurement schema which allows to quantitatively estimating the influence that an activated strategy has on the KPIs used to measure each objective defined by each VO enterprise (val_Sis_KPIixk). For more detailed description in terms of equations that define the behaviour of the system and the relationships of the variables depicted, we refer readers to [24].

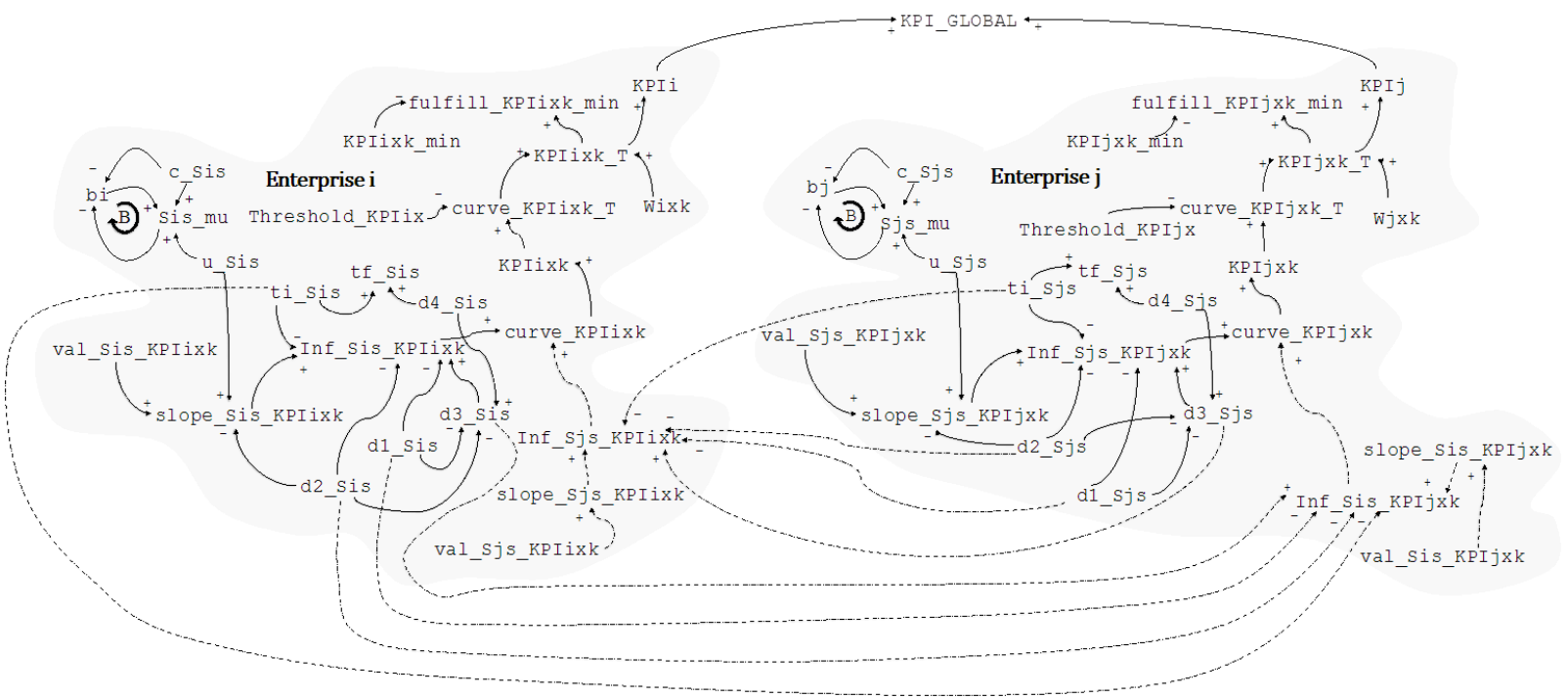

Figure 1: Causal loop diagram - strategies alignment process.

Table I: Elements of the system dynamics simulation model.

\begin{tabular}{|c|c|}
\hline Elements & Description \\
\hline $\mathrm{bi}$ & Budget reserved by the enterprise $i$ to invest in the activation of the strategies Sis \\
\hline C_Sis & Cost of the strategy \\
\hline curve KPIixk & Function that models the increase of KPIixk considering all the activated strategies \\
\hline curve KPIixk T & Function that models the increase of KPIixk when Threshold_KPIixk value is computed \\
\hline d1_Sis & Delay time of activation of the strategy $s$ in enterprise $i$ Sis \\
\hline d2_Sis & $\begin{array}{l}\text { Time between the Sis starts to influence the KPIixk until the maximum level of influence in } \\
\text { is achieved }\end{array}$ \\
\hline d3 Sis & Time period in which Sis is exerting the highest influence (val_Sis_KPIixk) on the KPIixk \\
\hline d4 Sis & Total duration of Sis \\
\hline fulfill KPIixk min & Minimum increase that the enterprise estimates for the KPIixk \\
\hline Inf_Sis_KPIixk & Function that models the behaviour of the KPIixk when Sis is activated \\
\hline KPI_GLOBAL & Increase experienced $K P I$ defined at network level \\
\hline KPI i & Increase experienced by the $K P I$ defined at enterprise level \\
\hline KPIixk & $\begin{array}{l}\text { Increase observed in the KPIixk when the Sis is activated: Sis activated in the same enterprise } \\
\text { (intra-enterprise) and Sjs activated by other enterprises (inter-enterprise) }\end{array}$ \\
\hline KPIixk_min & Minimum increase that the enterprise estimates for the KPIixk \\
\hline KPIixk T & Increase experienced by the KPIixk once the Threshold_KPIixk is computed \\
\hline Sis mu & Monetary units invested in the activation of Sis \\
\hline slope_Sis_KPIikx & Slope of the ramp in represented in curve_KPIixk \\
\hline tf $\mathrm{Sis}$ & Ending time for the of Sis \\
\hline Threshold KPIixk & Value from which the associated KPIixk is affected by the activation of a strategy Sis \\
\hline tisis & Initial time of activation of Sis \\
\hline u_sis & Units of strategy $[u . s]$ Sis to be activated \\
\hline val_Sis_KPIixk & $\begin{array}{l}\text { Value that registers the increase or decrease of the KPIixk when one unit of Sis is activated } \\
\text { (u_Sis) }\end{array}$ \\
\hline Wikx & Relevance that the KPIixk has for enterprise $i$ \\
\hline
\end{tabular}




\subsection{Method to solve the strategies alignment process}

A method to support the VO planner in the process of selecting partners, based on the strategies alignment among the potential candidates to the VBE, is proposed, so that the enterprises with higher levels of alignment will be those likely to be selected with the aim of forming a stable and sustainable VO. Eight are the phases that make up the proposed methodology:

Phase 1. Identification of suitable candidate enterprises from the VBE, taking into account their competencies; and roles identification: VO planner, and Enterprises Manager.

Phase 2. Definition of key performance indicators (KPIs). Each suitable candidate enterprise (identified in the phase 1) defines its own objectives and the data used to measure the level of achievement of such objectives is gathered (fulfill_KPIixk_min, KPIixk, KPIixk_min, Threshold_KPIixk, Wikx).

Phase 3. Each suitable candidate enterprise formulates the business strategies with the main aim of reaching its own defined objectives. The data items, associated to the strategies are determined (bi, c_Sis, d1_Sis, d2_Sis, d4_Sis, tf_Sis).

Phase 4. Estimation of the Influence Values. When a strategy Sis is activated, the defined objectives "receive" positive or negative influences, thus increasing or decreasing the level of the KPIixk. The parameter val_Sis_KPIixk models the influences that the activation of a specific strategy Sis has on a defined KPIixk. Intra-enterprise influences are modelled through val_Sis_KPIixk and val_Sjs_KPIjxk, whilst inter-enterprise influences are represented by val_Sis_KPIjxk and val_Sjs_KPIixk.

Phase 5. Uploading the data in a database management system, which stores the information required to feed the strategies alignment model, built in the system dynamicssimulation model. In our implementation, a Microsoft Access Database is specifically designed.

Phase 6. Automatic creation of the Strategies Alignment Model. The Strategies Alignment GENerator (SAGEN) is a tool that automatically generates the strategies alignment model according to the SD simulation software. A database, built in Microsoft Access, contains the necessary input information to automatically build the strategies alignment model in the SAGEN tool. SAGEN generates an XML file containing the parameters and the structure required in SD to build the strategies alignment model for the simulation software [24].

Phase 7. Generate solutions. The optimisation module, which is integrated in the systemdynamics simulation software, generates the solutions for the decision variables $u \_S i s$ (units of strategies to activate) and $t i$ Sis (the range of time in which to initiate the activation of a strategy) that optimise the network performance, i.e. maximising KPI_GLOBAL.

Phase 8. Solutions Assessment. VO planner focuses on the enterprises whose strategies are aligned, being these ones the partners to be potentially selected for the formation of a stable and sustainable VO.

\subsection{Integrated Environment}

In this section an integrated software environment is proposed to facilitate the calculation and assessment of the system dynamics simulation-based model proposed to deal with the strategies alignment process. A simulation tool is included in this integrated environment to automatically solve the proposed system-dynamics simulation based model, assessing and supporting the strategies alignment process. AnyLogic simulation software [28] is selected as a development software, since it allows representing, in SD, the elements of the strategies alignment model. Moreover, the models constructed in AnyLogic have the particularity of being read from XML (Extensible Markup Language). The integrated environment includes a 
database management system to gather all data required for building the strategies alignment system dynamics model. Finally, the integrated environment also includes a component to automatically generate the simulation model for the strategies alignment: the Strategies Alignment model GENerator (SAGEN) [24]. The procedure followed in SAGEN is to: (i) collect all the required data to feed the strategies alignment simulation model in the database management system; (ii) establish a OCDBConnection; (iii) create an XML file with SAGEN, which includes all the elements necessary to represent the strategies alignment model in system-dynamics; (iv) build both the flow diagram of the strategies alignment simulation model, and the optimization experiment, in the AnyLogic simulation software [28]. AnyLogic integrates both simulation and optimisation experiments. Through optimisation techniques, AnyLogic searches the values of the model parameters (decision variables) that lead to obtain greater performance levels of the strategies alignment system dynamics model, given an objective function that consists of the maximisation of the GLOBAL_KPI, and the set of constraints. The optimisation module is used jointly with the simulation module to carry out the parameters search. AnyLogic uses OptQuest engine to perform the optimisation of the simulation model [29]. The use of the integrated environment allows the VO planner to solve and assess the strategies alignment process from a collaborative perspective. The described strategies alignment simulation model (system dynamic based), jointly with the integrated environment, supports the VO planner regarding the selection of partners whose strategies have higher levels of strategies alignment, dealing with misalignments, and reducing potential conflicts in the generated VO. For more detailed description of the decision-support tool to deal with the strategies alignment process, we refer readers to [30].

\section{APPLICATION CASE}

The proposed use case consists of a VBE with ten enterprises. This case is a real simplified example case from building industry. A collaborative operation in construction industry is exemplified, providing market opportunities to smaller organisations, which will benefit from higher profits and greater chances of survivability in current globalised and highly dynamic markets. The practical exercise performed in this use case has provided useful results and served as a proof of concept to validate the strategies alignment simulation-based approach. In this specific use case, a new business opportunity is identified for the construction of ecofriendly buildings, which requires the use of novel technology, materials, expertise and capabilities from various building disciplines. The VO planner identifies the competencies required to create the $\mathrm{VO}$ and materialises this business opportunity. For the constitution of the VO a set of competencies (C), identified by the VO planner, are needed. These competencies are summarised: C1 - Architecture; C2 - Construction Technology; C3 Landscaping; C4 - Construction Services (Plumbing, Electricity and Heating); C5 - Surveyor; and C6 - Materials provision. The VO planner knows the competencies of each enterprise belonging to the VBE, as this information is given by each of the enterprises when joining the VBE. They provide such information as they will benefit from the VBE by participating in new business opportunities from a collaborative perspective, allowing them to better achieve common or compatible goals that could not be achieved by isolate entities. For this example, let us consider that the competencies required by the VO are only found in four of the enterprises belonging to the VBE. Based on the needed competencies, the VO planner reduces the space of options to create the $\mathrm{VO}$; thus, four enterprises out of the ten enterprises belonging to the VBE are identified. As it can be seen in Table II, each of the four enterprises has part of the necessary competencies to structure the VO. In order to guarantee that all the competencies defined by the VO planner are met, so that the VO can be constituted, seven sets of feasible combinations of enterprises (E) are proposed: (i) $\operatorname{set}_{1}$ : $E_{1}, E_{4}, E_{7}, E_{8}$; (ii) set 2 : 
$E_{1}, E_{4}, E_{7}$; (iii) set se $_{1}, E_{4}, E_{8}$; (iv) set 4 : $E_{1}, E_{7}, E_{8}$; (v) set : $_{4}, E_{7}, E_{8}$; (vi) set 6 : $E_{1}, E_{8}$; (vii) set 7 : $\mathrm{E}_{4}, \mathrm{E}_{7}$. The proposed combinations meet the premise that among all the enterprises, which join to collaborate and form the VO, gather all the necessary competencies to cover the needed requirements. Next, the VO planner proceeds to select the best set of enterprises, and starts the partners' selection process. The selection of VO partners is supported by the proposed strategies alignment model and the integrated environment. In order to carry out the partners' selection process, the VO planner also knows the objectives and strategies defined by each of the enterprises belonging to the VBE. In this use case, each enterprise defines three objectives to carry out the new business opportunity (construction of eco-friendly buildings); the achievement of the objectives is measured through three KPIs. Let us also assume that in order to achieve the defined objectives; each enterprise formulates four business strategies, for carrying out the specific business opportunity. The enterprises have a certain budget to carry out these strategies. Moreover, the enterprises define that the level of performance to be achieved by each defined KPI must be higher than 0 . The data structure, of the formulated objectives and the defined strategies, is provided in Table II.

Table II: SAM data structure.

\begin{tabular}{|c|c|c|c|c|c|c|c|}
\hline E1 & b1 & Compe & encies: C & , C2, C3 & & & \\
\hline u_S11 & c_S11 & d1_S11 & d2_S11 & d4_S11 & KPI111 & KPI121 & KPI131 \\
\hline u_S12 & c_S12 & d1_S12 & d2_S12 & d4_S12 & Threshold_KPI111 & Threshold_KPI121 & Threshold_KPI131 \\
\hline u_S13 & c_S13 & d1_S13 & d2_S13 & d4_S13 & W111 & W121 & W131 \\
\hline u_S14 & c_S14 & d1_S14 & d2_S14 & d4_S14 & val_Sis_KPI111 & val_Sis_KPI121 & val_Sis_KPI131 \\
\hline E4 & b4 & Compe & encies: C & $, \mathrm{C2}, \mathrm{C} 4$ & & & \\
\hline u_S41 & c_S41 & d1_S41 & d2_S41 & d4_S41 & KPI411 & KPI421 & KPI431 \\
\hline u_S42 & c_S42 & d1_S42 & d2_S42 & d4_S42 & Threshold_KPI411 & Threshold_KPI421 & Threshold_KPI431 \\
\hline u_S43 & c_S43 & d1_S43 & d2_S43 & d4_S43 & W411 & W421 & W431 \\
\hline u_S44 & c_S44 & d1_S44 & d2_S44 & d4_S44 & val_Sis_KPI411 & val_Sis_KPI421 & val_Sis_KPI431 \\
\hline E7 & b7 & Compe & encies: C & , C5, C6 & & & \\
\hline u_S71 & c_S71 & d1_S71 & d2_S71 & d4_S71 & KPI711 & KPI721 & KPI731 \\
\hline u_S72 & c_S72 & d1_S72 & d2_S72 & d4_S72 & Threshold_KPI711 & Threshold_KPI721 & Threshold_KPI731 \\
\hline u_S73 & c_S73 & d1_S73 & d2_S73 & d4_S73 & W711 & W721 & W731 \\
\hline u_S74 & c_S74 & d1_S74 & d2_S74 & d4_S74 & val_Sis_KPI711 & val_Sis_KPI721 & val_Sis_KPI731 \\
\hline E8 & b8 & Compe & encies: C & $, \mathrm{C5}, \mathrm{C6}$ & & & \\
\hline u_S81 & c_s81 & d1_S81 & d2_S81 & d4_S81 & KPI811 & KPI481 & KPI831 \\
\hline u_S82 & c_s82 & d1_S82 & d2_S82 & d4_S82 & Threshold_KPI811 & Threshold_KPI821 & Threshold_KPI831 \\
\hline u_S83 & c_s83 & d1_S83 & d2_S83 & d4_S83 & W811 & W821 & W831 \\
\hline u_S84 & c_s84 & d1_S84 & d2_S84 & d4_S84 & val_Sis_KPI811 & val_Sis_KPI821 & val_Sis_KPI831 \\
\hline
\end{tabular}

In the process of selecting the suitable set of enterprises for structuring the $\mathrm{VO}$, the VO planner applies the strategies alignment filter, which is an approach based on the selection of partners whose strategies, formulated to deal with the identified new business opportunity, are more aligned. For this purpose, the SAM and the integrated environment proposed are used. The VO planner computes, for each set of enterprises (i.e. each potential VO configuration), the SAM in the integrated environment (system dynamics simulation software). The optimisation of the SAM results on the strategies to activate $\left(u \_S i s\right)$ by each enterprise belonging to each potential set (according to the owned budget). The resulting strategies are characterised by being aligned. In addition, the SAM provides the time in which to activate the proposed strategies, $t i \_S i s$, given in units of time related to the overall length of time corresponding to the life cycle of the VO. Each identified set of enterprises corresponds to a different scenario of optimisation and simulation. The modelled and simulated scenarios generate different performance results at enterprise (KPIi) and network level (KPI_GLOBAL), 
depending on the strategies to activate, as a result of the optimisation experiment. In the light of this, the results of the optimisation and simulation experiments carried out in the SAM and the integrated environment are presented (Table III). In order to compare the KPIs at enterprise and network level, between each of the sets, a normalised Eq. (1) has been applied. The normalised KPI_GLOBAL' has been computed as a result of the average of $K P I_{i}^{\prime}$.

$$
K P I_{i}^{\prime}=\frac{\left(\frac{\sum_{i} K P I_{i}}{k}\right)}{K P I_{i}} \text {, where } k \text { is the total number of sets }
$$

The combination of enterprises with higher level of performance and therefore with higher level of alignment, will be the ones proposed to the VO planner to create the VO. In this case study belonging to the building industry, set 5 , which consists of $\mathrm{E}_{4}, \mathrm{E}_{7}$ and $\mathrm{E}_{8}$, is the first proposed combination of enterprises to be selected for the VO establishment. The strategies to activate are $u \_S 44: 3,3$ units of strategies ( $\left.t i \_S 44: 0,123\right) ; u \_S 71: 2,5$ ( $t i \_S 71$ : 0,049); $u \_S 81$ : 0,8 (ti_S34: 0,051) and $u_{-} S 82: 1$ ( $\left.t i_{-} S 82: 0,083\right)$, resulting in the highest normalised performance level KPI_GLOBAL': 1,477 (see Table III). The VO planner will take into consideration set $_{1}$ and set $_{6}$ as second and third options; using the obtained KPI_GLOBAL' as quantitative value for ranking the set of partners to be selected in the creation of a stable VO.

Table III: Application - SAM solution.

\begin{tabular}{|c|c|c|c|c|c|c|c|}
\hline & set $_{1}$ & set $_{2}$ & set $_{3}$ & set $_{4}$ & set $_{5}$ & set $_{6}$ & set $_{7}$ \\
\hline u_S11 (ti_S11) & $0(0)$ & $0(0)$ & $0(0)$ & $0,6(0)$ & - & $1,2(0)$ & - \\
\hline$u \_S 12\left(t i \_S 12\right)$ & $0(0)$ & $0(0)$ & $0(0)$ & $1(0)$ & - & $0(0)$ & - \\
\hline$u \_S 13\left(t i \_S 13\right)$ & $2(0,073)$ & 0 & $0(0)$ & $1(0,084)$ & - & $0(0)$ & - \\
\hline$u \_S 14\left(t i \_S 14\right)$ & $1,5(0,102)$ & 2,5 & 2,5 & $0,3(0,094)$ & - & $1,6(0,118)$ & - \\
\hline$u \_S 41\left(t i \_S 41\right)$ & $0(0)$ & $0(0)$ & $0(0)$ & - & $0(0)$ & - & $0(0)$ \\
\hline$u \_S 42\left(t i \_S 42\right)$ & $0(0)$ & $0(0)$ & $0(0)$ & - & $0(0)$ & - & $0(0)$ \\
\hline$u \_S 43\left(t i \_S 43\right)$ & $0(0)$ & $0(0)$ & $0(0)$ & - & $0(0)$ & - & $0(0)$ \\
\hline$u \_S 44\left(t i \_S 44\right)$ & $0,3(0,106)$ & $3(0,081)$ & $0,7(0,115)$ & - & $3,3(0,123)$ & - & $3,3(0,122)$ \\
\hline$u \_S 71\left(t i \_S 71\right)$ & $2,5(0,022)$ & $0,1(0)$ & - & $0,9(0)$ & $2,5(0,049)$ & - & $0,1(0,005)$ \\
\hline$u \_S 72\left(t i \_S 72\right)$ & $0(0)$ & $0(0)$ & - & $1(0,201)$ & $0(0)$ & - & $0(0)$ \\
\hline$u \_S 73\left(t i \_S 73\right)$ & $0(0)$ & $0(0)$ & - & $0(0)$ & $0(0)$ & - & $0(0)$ \\
\hline$u \_S 74\left(t i \_S 74\right)$ & $0(0)$ & $3,2(0,23)$ & - & $0,4(0)$ & $0(0)$ & - & $3,2(0,053)$ \\
\hline$u \_S 81\left(t i \_S 81\right)$ & $0,8(0,006)$ & - & $0,8(0,062)$ & $0,8(0,255)$ & $0,8(0,051)$ & $1,6(0,047)$ & - \\
\hline$u \_S 82\left(t i \_S 82\right)$ & $1(0,071)$ & - & $1(0,064)$ & $1(0,076)$ & $1(0,083)$ & $0,8(0,096)$ & - \\
\hline$u \_S 83\left(t i \_S 83\right)$ & $0(0)$ & - & $0(0)$ & $0(0)$ & $0(0)$ & $0(0)$ & - \\
\hline$u \_S 84\left(t i \_S 84\right)$ & $0(0)$ & - & $0(0)$ & $0(0)$ & $0(0)$ & $0(0)$ & - \\
\hline $\mathrm{KPI}_{1}$ & 1,266 & 0,126 & 1,194 & 0,928 & - & 1,485 & - \\
\hline $\mathrm{KPI}_{4}{ }^{\prime}$ & 0,130 & 1,143 & 0,322 & - & 1,849 & - & 1,557 \\
\hline $\mathrm{KPI}_{7}{ }^{\prime}$ & 1,679 & 0,875 & - & 0,936 & 0,961 & - & 0,549 \\
\hline$K P I_{8}{ }^{\prime}$ & 1,725 & - & 0,714 & 0,943 & 0,857 & 0,760 & - \\
\hline KPI_GLOBAL, & 1,200 & 0,715 & 0,743 & 0,936 & 1,222 & 1,123 & 1,053 \\
\hline
\end{tabular}

The application of SAM and the integrated environment, in a real use case of the building industry, has enabled to identify limitations associated to the proposed approach. The drawbacks identified are related with the data collection, required for the validation of the strategies alignment approach, used as a novel filter to assess partners selected for a VO. Nevertheless, in the application of the strategies alignment model, when creating a VO that fits the new business opportunity of constructing eco-friendly buildings, the four enterprises of the VBE, and the VO planner have observed that (i) the process of gathering the data required for feeding the SAM is relatively easy with the help of the VO planner, (ii) the level of understanding of the results generated is affordable, (iii) the results obtained from applying the SAM are consistent, and (iv) the improvement in network performance achieved is very relevant, so that the set of selected enterprises favour the creation of sustainable and stable $\mathrm{VO}$, reducing the potential misalignments during the VO operation. 


\section{CONCLUSIONS}

A strategies alignment simulation-based approach is proposed as a complementary step to the partners' selection process for a $\mathrm{CN}$. Concretely, the presented approach is addressed in the context of a VBE that enables the creation of VOs, as one type of CN. The partners' selection process of a VO starts with the selection of enterprises that have the proper competencies in order to guarantee that all the competencies defined by the VO planner are met. In addition to this requirement, the consideration of other criteria is necessary to create a stable and sustainable VO. The literature review has allowed identifying examples of other criteria (filters) such as the trust assessment and value systems alignment. Further to these filters, this paper proposes a novel approach based on the assessment of the strategies alignment, to deal with the partners' selection process when forming a VO. For this purpose, a system dynamics model, SAM, and an integrated environment to automatically compute the level of alignment of the enterprises belonging to a network are proposed. The degree of alignment is assessed through the calculation of the performance at enterprise and network level. The adopted performance approach provides, to the VO planner, a quantitative decision-making tool to quickly identify partners with aligned strategies. The ease of use is one of the advantages of the proposed approach, although when validating it in real networks, the collection of suitable and feasible data becomes an arduous task. As further work, each enterprise of the network could be represented as an agent that will behave according to the SD model defined to represent the strategies alignment process, at the micro level; i.e., modelling a multi-agent system (MAS) simulation approach. The outputs generated in one agent will be the inputs to the other agents representing the enterprises of the network. Motivated by this idea, a multimethod approach, using both SD and MAS, will be considered in future work. As the used simulation tool, AnyLogic, supports both simulation approaches, this extension of the model to a distributed perspective could be usefully performed.

\section{ACKNOWLEDGEMENT}

This work has been funded in part by Programa Val $\mathrm{i}+\mathrm{d}$ para investigadores en formación (ACIF 2012) and by the Uninova - Center of Technology and Systems and the Portuguese FCT-PEST program UID/EEA/00066/2013.

\section{REFERENCES}

[1] Poler, R. (Ed.); Carneiro, L. M.; Jasinski, T.; Zolghadri, M.; Pedrazzoli, P. (2012). Intelligent Non-hierarchical Manufacturing Networks, Networks and Telecommunications Series, ISTE Ltd. and John Wiley \& Sons, Hoboken

[2] Bititci, U. S.; Martinez, V.; Albores, P.; Parung, J. (2004). Creating and managing value in collaborative networks, International Journal of Physical Distribution \& Logistics Management, Vol. 34, No. 3/4, 251-268, doi:10.1108/09600030410533574

[3] Camarinha-Matos, L. M.; Afsarmanesh, H. (2005). Collaborative networks : a new scientific discipline, Journal of Intelligent Manufacturing, Vol. 16, No. 4-5, 439-452, doi:10.1007/s10845$\underline{005-1656-3}$

[4] Andres, B.; Poler, R. (2016). Models, guidelines and tools for the integration of collaborative processes in non-hierarchical manufacturing networks: a review, International Journal of Computer Integrated Manufacturing, Vol. 29, No. 2, 166-201, doi:10.1080/0951192X.2014. 1003148

[5] Afsarmanesh, H.; Camarinha-Matos, L. M. (2005). A framework for management of virtual organization breeding environments, Camarinha-Matos, L. M.; Afsarmanesh, H.; Ortiz, A. (Eds.), Collaborative Networks and Their Breeding Environments, PRO-VE 2005, IFIP - The International Federation for Information Processing book series, Vol. 186, Springer, Boston, 3548, doi: $10.1007 / 0-387-29360-4 \_4$ 
[6] Afsarmanesh, H.; Camarinha-Matos, L. M.; Msanjila, S. S. (2009). On management of $2^{\text {nd }}$ generation virtual organizations breeding environments, Annual Reviews in Control, Vol. 33, No. 2, 209-219, doi:10.1016/j.arcontrol.2009.05.007

[7] Huang, B.; Gao, C.; Chen, L. (2011). Partner selection in a virtual enterprise under uncertain information about candidates, Expert Systems with Applications, Vol. 38, No. 9, 11305-11310, doi:10.1016/j.eswa.2011.02.180

[8] Shamsuzzoha, A. H. M.; Kankaanpaa, T.; Helo, P.; Carneiro, L. M.; Almeida, R.; Fornasiero, R. (2010). Non-hierarchical collaboration in dynamic business communities, Camarinha-Matos, L. M.; Boucher, X.; Afsarmanesh, H. (Eds.), Collaborative Networks for a Sustainable World, PROVE 2010, IFIP Advances in Information and Communication Technology book series, Vol. 336, Springer, Berlin, 609-618, doi:10.1007/978-3-642-15961-9 73

[9] Camarinha-Matos, L. M.; Afsarmanesh, H. (2007). A framework for virtual organization creation in a breeding environment, Annual Reviews in Control, Vol. 31, No. 1, 119-135, doi:10.1016/j.arcontrol.2007.03.006

[10] Camarinha-Matos, L. M.; Oliveira, A. I.; Sesana, M.; Galeano, N.; Demsar, D.; Baldo, F.; Jarimo, T. (2009). A framework for computer-assisted creation of dynamic virtual organisations, International Journal of Production Research, Vol. 47, No. 17, 4661-4690, doi:10.1080/ 00207540902847272

[11] Rosas, J.; Macedo, P.; Camarinha-Matos, L. M. (2011). Extended competencies model for collaborative networks, Production Planning \& Control, Vol. 22, No. 5-6, 501-517, doi: $10.1080 / 09537287.2010 .536622$

[12] Msanjila, S. S.; Afsarmanesh, H. (2010). FETR: a Framework to establish trust relationships among organizations in VBEs, Journal of Intelligent Manufacturing, Vol. 21, No. 3, 251-265, doi:10.1007/s10845-008-0178-1

[13] Macedo P.; Camarinha-Matos, L. M. (2013). A qualitative approach to assess the alignment of Value Systems in collaborative enterprises networks, Computers \& Industrial Engineering, Vol. 64, No. 1, 412-424, doi:10.1016/j.cie.2012.09.019

[14] Andres, B.; Poler, R. (2015). Dealing with the alignment of strategies within the collaborative networked partners, Camarinha-Matos, L. M.; Baldissera, T.; Di Orio, G.; Marques, F. (Eds.), Technological Innovation for Cloud-Based Engineering Systems, DoCEIS 2015, IFIP Advances in Information and Communication Technology book series, Vol. 450, Springer, Cham, 13-21, doi:10.1007/978-3-319-16766-4_2

[15] Mejia, R.; Molina, A. (2002) Virtual enterprise broker: Processes, methods and tools, CamarinhaMatos, L. M. (Ed.), Collaborative business ecosystems and virtual enterprises, IFIP - The International Federation for Information Processing book series, Vol. 85, Springer, Boston, 8190, doi:10.1007/978-0-387-35585-6_10

[16] Junior, O. C. A.; Rabelo, R. J. (2013). A KPI model for logistics partners' search and suggestion to create virtual organisations, International Journal of Networking and Virtual Organisations, Vol. 12, No. 2, 149-177, doi:10.1504/IJNVO.2013.053746

[17] Oliveira, A. I.; Shafahi, M.; Afsarmanesh, H.; Ferrada, F.; Camarinha-Matos, L. M. (2016). Competence matching in collaborative consortia for service-enhanced products, Afsarmanesh, H.; Camarinha-Matos, L. M.; Lucas Soares, A. (Eds.), Collaboration in a Hyperconnected World, PRO-VE 2016, IFIP Advances in Information and Communication Technology book series, Vol. 480, Springer, Cham, 350-360, doi:10.1007/978-3-319-45390-3_30

[18] Crispim, J. A.; de Sousa, J. P. (2010). Partner selection in virtual enterprises, International Journal of Production Research, Vol. 48, No. 3, 683-707, doi:10.1080/00207540802425369

[19] Macedo, P.; Abreu, A.; Camarinha-Matos, L. M. (2010). A method to analyse the alignment of core values in collaborative networked organisations, Production Planning \& Control, Vol. 21, No. 2, 145-159, doi:10.1080/09537280903441930

[20] Sarkis, J.; Talluri, S.; Gunasekaran, A. (2007). A strategic model for agile virtual enterprise partner selection, International Journal of Operations \& Production Management, Vol. 27, No. 11, 1213-1234, doi:10.1108/01443570710830601

[21] Li, M.; Wu, C.; Zhang, L.; You, L.-N. (2015). An intuitionistic fuzzy-TODIM method to solve distributor evaluation and selection problem, International Journal of Simulation Modelling, Vol. 14, No. 3, 511-524, doi:10.2507/IJSIMM14(3)CO12 
[22] Angulo, P. S.; De Benito Martín, J. J.; Araúzo, J. A.; del Olmo Martínez, R. (2005). Partner selection in dynamic virtual enterprises using multi-agent systems, Proceedings of the International Conference of Artificial Intelligence (IC-AI'05), 450-456

[23] Camarinha-Matos, L. M.; Oliveira, A. I.; Ferrada, F.; Sobotka, P.; Vatascinova, A.; Thamburaj, V. (2015). Collaborative enterprise networks for solar energy, Proceedings of the International Conference on Computing and Communication Technologies (ICCCT 2015), 93-98, doi: $10.1109 /$ ICCCT2.2015.7292726

[24] Andres, B.; Poler, R. (2016). A decision support system for the collaborative selection of strategies in enterprise networks, Decision Support Systems, Vol. 91, 113-123, doi:10.1016/ j.dss.2016.08.005

[25] Andres, B.; Macedo, P.; Camarinha-Matos, L. M.; Poler, R. (2014). Achieving coherence between strategies and value systems in collaborative networks, Camarinha-Matos, L. M.; Afsarmanesh, H. (Eds.), Collaborative Systems for Smart Networked Environments, PRO-VE 2014, IFIP Advances in Information and Communication Technology book series, Vol. 434, Springer, Berlin, 261-272, doi:10.1007/978-3-662-44745-1 26

[26] Forrester, J. W. (1961). Industrial dynamics, MIT Press, Cambridge

[27] Campuzano, F.; Mula, J. (2011). Supply Chain Simulation. A System Dynamics Approach for Improving Performance, Springer, London

[28] The AnyLogic Company. AnyLogic, from http://www.anylogic.com/, accessed on 10-10-2015

[29] Kleijnen, J. P. C.; Wan, J. (2007). Optimization of simulated systems: OptQuest and alternatives, Simulation Modelling Practice and Theory, Vol. 15, No. 3, 354-362, doi:10.1016/j.simpat. 2006.11.001

[30] Andres, B.; Poler, R.; Rosas, J.; Camarinha-Matos, L. M. (2016). A decision-support tool to deal with the strategies alignment process in collaborative networks, Camarinha-Matos, L. M., Falcão, A. J.; Vafaei, N.; Najdi, S. (Eds.), Technological Innovation for Cyber-Physical Systems, DoCEIS 2016, IFIP Advances in Information and Communication Technology book series, Vol. 470, Springer, Cham, 3-10, doi:10.1007/978-3-319-31165-4 1 